Contents List available at RAZI Publishing

Matriks Sains Matematik (MSMK)

Journal Homepage: http://www.razipublishing.com/journals/matriks-sains-matematik https://doi.org/10.26480/msmk.01.2017.01.08

\title{
Existence and Uniqueness of Common Fixed Point for Mappings Satisfying Integral Type Contractive Conditions in G-Metric Spaces
}

\author{
Muhammad Shoaib1, Muhammad Sarwar ${ }^{1}$, Sultan Hussain ${ }^{2}$ and Gohar Ali ${ }^{3}$ \\ ${ }^{1}$ Department of Mathematics, University of Malakand, Chakdara Dir(L), Pakistan \\ 2Department of Mathematics, Comsats Institute of Information Technology, Abbottabad, Pakistan
}

3Department of Mathematics, Islamia College University of Peshawar, Pakistan*Corresponding author email: sarwarswati@gmail.com

This is an open access article distributed under the Creative Commons Attribution License, which permits unrestricted use, distribution, and reproduction in any medium, provided the original work is properly cited

\section{ARTICLE DETAILS}

Article history:

Received 12 August 2016 Accepted 12 December 2016

Available online 20 January 2017

Keywords:

Common Fixed Point, Complete

G-metric Space, Integral Type

Contraction.

\section{ABSTRACT}

We establish common fixed point results for two self-mappings satisfying integral type contractive conditions in the framework of complete G-metric spaces. The established results generalize and extend many results in the existing literature. Moreover, we provide some comparative examples to show that our results are substantial improvements of some known results in literature.

\section{INTRODUCTION}

Fixed point theory is one of the most active research subject in the development of nonlinear analysis. It is used in pure and applied mathematics and play a key part in nonlinear functional analysis as well as used for proving the existence theorems for nonlinear differential and integral equations. One of the main result in this area is the Banach contraction principle proved by Banach, which says that any contraction self-mappings on a complete metric space has a unique fixed point Because of its importance for mathematical theory, this Principle has been extended and generalized in many directions and the references cited therein (Abbas et al., 2011; Suzuki, 2008; Zamfrescu, 1972).

In 2002, an integral version of the Banach fixed point theorem (Branciari, 2002). This result was more generalized and extended by many authors for the existence of fixed points and common fixed points for numerous mapping satisfying integral type contraction (Aliouche, 2006; Dsoudi and Merghadi, 2008; Khojesteh et al., 2010; Rhoades, 2003; Suuki, 2006; Liu and Kang, 2000). In recent years, there has been increasing interest in the study of fixed points of mappings satisfying integral type contraction. Recently, a studied common fixed point theorem for integral type contraction in generalized metric spaces (Ayadi, 2012).

The aims of this manuscript is to study the existence and uniqueness of common fixed points for contractive mappings of integral type in the set-up of generalized metric spaces. Our results generalize the G-metric spaces and carry the results in metric spaces to G-metric spaces (Ayadi, 2012; Liu et al., 2011). Throughout this paper $N$ denotes the set of positive integers, $R$ is the set of real numbers, $R^{+}$is the set of nonnegative real numbers and

$$
\Phi=\left\{\begin{array}{l}
\phi \mid \phi: \mathrm{R}^{+} \rightarrow \mathrm{R}^{+} \text {where } \phi \text { is Lebesgue integrable, summable } \\
\text { on each compact subset of } \mathrm{R}^{+} \text {and } \int_{0}^{\varepsilon} \phi(t) d t>0 \text { for each } \varepsilon>0
\end{array}\right\}
$$

1. Preliminaries

Before going to the main results, we recall some definitions and results from the existing literature.

Definition 2.1 (Manro et al., 2013; Mustafa and Sims, 2006). Let $X$ be a non-empty set and let $G: X \times X \times X \rightarrow \mathrm{R}^{+}$be a function satisfying the following conditions:

1) $G(x, y, z)=0$ implies that $x=y=z$ for all $x, y, z \in X$;

2) $0<G(x, x, y)$ for all $x, y, \in X$ with $x \neq y$;

3) $G(x, x, y) \leq G(x, y, z)$ for all $x, y, z \in X$ with $y \neq z$;

4) $\quad G(x, y, z)=G(x, z, y)=G(y, z, x)=\cdots$ (symmetry in all three variables);

5) $G(x, y, z) \leq G(x, a, a)+G(a, y, z)$ for all $x, y, z, a \in X$

Then it is called G-metric on $X$ and the pair $(X, G)$ is called Gmetric space.

Example 2.2. Let $X=[0, \infty)$ and $G: X \times X \times X \rightarrow \mathrm{R}^{+}$be the function defined as follow

$G(x, y, z)=\max \{|x-y|,|y-z|,|z-x|\} \quad$ for all $\quad x, y, z \in X$.

Then $G$ is G-metric on $X$.

Proposition 2.3 (Mustafa and Sims, 2006). Let $(X, G)$ be a Gmetric space. The following are equivalent:

1) $\boldsymbol{Q}_{n} \bullet$ is $\mathrm{G}$-convergent to $\mathrm{X}$;

2) $\quad G\left(x_{n}, x_{n}, x\right) \rightarrow 0$ as $n \rightarrow \infty$

3) $\quad G\left(x_{n}, x, x\right) \rightarrow 0$ as $n \rightarrow \infty$

4) $\quad G\left(x_{n}, x_{m}, x\right) \rightarrow 0$ as $n, m \rightarrow \infty$ 
Proposition 2.4 (Sintunavarat and Kumam., 2012) Let $f$ and $g$ be weakly compatible self-mappings on a set $X$. If $f$ and $g$ have a unique point of coincidence $\xi=f \psi=g \psi$, then $\xi$ is the unique common fixed point of $f$ and $g$.

Proposition 2.5 (Sintunavarat and Kumam., 2012) Let $(X, G)$ be a Gmetric space. Then following statement holds:

1) $\quad|G(x, y, z)-G(x, y, a)| \leq \max \{G(a, z, z), G(z, a, a)\}$,

2) $\quad G(x, y, y) \leq 2 G(y, x, x)$.

Lemma 2.6 (Liu et al., 2011). Let $\phi \in \Phi$ and $\left\{r_{n}\right\}_{n \in \mathrm{N}}$ is a non-negative sequence with $\lim _{n \rightarrow \infty} r_{n}=a$. Then

$$
\lim _{n \rightarrow \infty} \int_{0}^{r_{n}} \phi(t) d t=\int_{0}^{a} \phi(t) d t .
$$

Lemma 2.7 (Liu et al., 2011). Let $\phi \in \Phi$ and $\left\{r_{n}\right\}_{n \in \mathrm{N}}$ is a non-negative sequence. Then

$$
\lim _{n \rightarrow \infty} \int_{0}^{r_{n}} \phi(t) d t=0 \Leftrightarrow \lim _{n \rightarrow \infty} r_{n}=0 .
$$

Definition 2.8 (Sintunavarat and Kumam, 2012). Let $S$ and $T$ be selfmappings of a nonempty set $X$.

1) A point $x \in X$ is said to be a fixed point of $T$ if $T x=x$.

2) A point $x \in X$ is said to be a coincidence point of $S$ and $T$ if $S x=T x$ and we call the point $w=S x=T x$ a point of coincidence of $S$ and $T$.

3) A point $x \in X$ is said to be a common fixed point of $S$ and $T$ if $x=S x=T x$.

Definition 2.9 (Abbas et al., 2011; Aliouche, 2006). Let $X$ be a nonempty set the mappings $T, f: X \rightarrow X$ are said to be weakly compatible if they commute at their coincidence point (i.e., $T f x=f T x$ whenever $T x=f x$.

Theorem 2.10. (Branclari, 2002). If $T$ be a self-map of a complete metric space $(X, d)$ such that for all $x, y \in X$

$\int_{0}^{d\left(T_{x}, T_{y}\right)} \phi(t) d t \leq \eta \int_{0}^{d(x, y)} \phi(t) d t, \eta \in(0,1)$.

where, $\phi \in \Phi$. Then $T$ has a unique fixed point in $X$.

In 2011, a researcher proved the following results which generalized Theorem 2.10 in metric spaces (Liu et al., 2011).

Theorem 2.11. If $T$ be a mapping from a complete metric space $(X, d)$ to itself such that for all $x, y \in X$

$$
\int_{0}^{d\left(T_{x}, T_{y}\right)} \phi(t) d t \leq \alpha(d(x, y)) \int_{0}^{d(x, y)} \phi(t) d t,
$$

where, $\quad \phi \in \Phi$ and $\alpha:[0, \infty) \rightarrow[0,1)$ is a function with $\limsup _{s \rightarrow t} \alpha(s)<1, \forall t>0$. Then $T$ has a unique fixed point in $X$.

Theorem 2.12. If $T$ be a mapping from a complete metric space $(X, d)$ to itself such that for all $x, y \in X$

$$
\int_{0}^{d(T x, T y)} \phi(t) d t \leq \alpha\left(d(x, y) \int_{0}^{d(x, T x)} \phi(t) d t+\beta\left(d(x, y) \int_{0}^{d\left(y, T_{y}\right)} \phi(t) d t,\right.\right.
$$

where, $\phi \in \Phi$ and $\alpha, \beta:[0,+\infty) \rightarrow[0,1)$ is a function with $\lim \sup _{s \rightarrow t} \frac{\alpha(s)}{1-\beta(s)}<1 \quad \forall t>0$. Then $T$ has a unique fixed point in $X$.

In 2014, generalized the above theorem for integral type contraction and proved the following theorem in metric spaces (Liu et al., 2014a).

Theorem 2.13. If $T$ be a mapping from a complete metric space $(X, d)$ to itself such that for all $x, y \in X$

$$
\begin{aligned}
\int_{0}^{d\left(T x, T_{y}\right)} \phi(t) d t & \leq \alpha(d(x, y)) \int_{0}^{d(x, T x)} \phi(t) d t+\beta\left(d(x, y) \int_{0}^{d\left(y, T_{y}\right)} \phi(t) d t\right. \\
& +\gamma\left(d(x, y) \int_{0}^{d(x, y)} \phi(t) d t,\right.
\end{aligned}
$$

For all $x, y \in X$ where, $\phi \in \Phi$ and $\alpha, \beta, \gamma:[0,+\infty) \rightarrow[0,1)$ is a function with

$$
\begin{gathered}
\alpha(t)+\beta(t)+\gamma(t)<1 \forall t \in \mathrm{R}^{+}, \\
\limsup [\alpha(s)+\beta(s)+\gamma(s)]<1 \forall t>0 .
\end{gathered}
$$

Then $T$ has a unique fixed point in $X$

Recently, a researcher has established the following common fixed point theorems in generalized metric spaces (Ayadi, 2012)
Theorem 2.14. Let $(X, G)$ be complete G-metric space and $f, g: X \rightarrow X$ be a mapping such that

$G(f x, f y, f z) \leq \alpha G(g x, g y, g z)$,

for all $x, y, z \in X$ where $\alpha \in[0,1)$. If $f(X) \subset g(X)$ and $g(X)$ is a complete subspace of $X$. Then $f$ and $g$ have a unique point of coincidence in $X$. Moreover if $f$ and $g$ are weakly compatible, then $f$ and $g$ have a unique common fixed point in $X$.

Theorem 2.15. Let $(X, G)$ be complete G-metric space and $f, g: X \rightarrow X$ be a mapping such that

$$
\int_{0}^{G(f x, f y, f z)} \phi(t) d t \leq \alpha \int_{0}^{(G(g x, g y, g z))} \phi(t) d t,
$$

for all $x, y, z \in X$ where $\alpha \in[0,1)$ and $\phi \in \Phi$. If $f(X) \subset g(X)$ and $g(X)$ is a complete subspace of $X$. Then $f$ and $g$ have a unique point of coincidence in $X$. Moreover if $f$ and $g$ are weakly compatible, then $f$ and $g$ have a unique common fixed point in $X$.

\section{Main results}

Theorem 3.1. Let $(X, G)$ be complete G-metric space and $f, g: X \rightarrow X$ be a mapping such that

$$
\int_{0}^{G(f x, f y, f z)} \phi(t) d t \leq \alpha(G(g x, g y, g z)) \int_{0}^{(G(g x, g y, g z))} \phi(t) d t,
$$

for all $x, y, z \in X$, where $\phi \in \Phi$ and $\alpha:[0,+\infty) \rightarrow[0,1)$ is a function with $\alpha(t)<1 \forall t \in \mathrm{R}^{+}, \quad \limsup \alpha(s)<1, \quad \forall t>0$. If $f(X) \subset g(X)$ and $g(X)$ is a complete subspace of $X$. Then $f$ and $g$ have a unique point of coincidence in $X$. Moreover if $f$ and $g$ are weakly compatible, then $f$ and $g$ have a unique common fixed point in $X$.

Proof. Let $x_{0}$ be arbitrary point in $X$. Since $f(X) \subset g(X)$ choose $x_{1} \in X$ such that $g x_{1}=f x_{0}$ choose $x_{2} \in X$ such that $g x_{2}=f x_{1}$ in last we choose $x_{n+1} \in X$ such that $y_{n}=g x_{n+1}=f x_{n}$. Let $G_{n}=G\left(y_{n}, y_{n+1}, y_{n+2}\right)$ by using (3.1) we get

$$
\begin{aligned}
\int_{0}^{G_{n}} \phi(t) d t & =\int_{0}^{G\left(x_{n}, f x_{n+1}, f x_{n+2}\right)} \phi(t) d t \\
& \leq \alpha\left(G\left(g x_{n}, g x_{n+1}, g x_{n+2}\right)\right) \int_{0}^{G\left(g x_{n}, g x_{n+1}, g x_{n+2}\right)} \phi(t) d t \\
& =\alpha\left(G\left(f x_{n-1}, f x_{n}, f x_{n+1}\right)\right) \int_{0}^{G\left(f x_{n-1}, f x_{n}, f x_{n+1}\right)} \phi(t) d t \\
& =\alpha\left(G_{n-1}\right) \int_{0}^{G_{n-1}} \phi(t) d t \\
& \leq \int_{0}^{G_{n-1}} \phi(t) d t, \forall n \in \mathrm{N}
\end{aligned}
$$

which yields that

$$
G_{n} \leq G_{n-1}, \forall n \in \mathrm{N} .
$$

Thus, the sequence $\left\{G_{n}\right\}_{n \in \mathrm{N}}$ is decreasing. Consequently, them exist a constant $C$ such that

$$
\lim _{n \rightarrow \infty} G_{n}=c .
$$

We claim that $c=0$. If $c>0$, then from (3.2) we have

$$
\int_{0}^{G_{n}} \phi(t) d t \leq \alpha\left(G_{n-1}\right) \int_{0}^{G_{n-1}} \phi(t) d t .
$$

Taking limit sup and using equation (3.4) and Lemma 2.6 we get

$$
\begin{aligned}
0<\int_{0}^{c} \phi(t) d t & =\limsup _{n \rightarrow \infty} \int_{0}^{G_{n}} \phi(t) d t \leq \limsup _{n \rightarrow \infty}\left(\alpha\left(G_{n-1}\right) \int_{0}^{G_{n-1}} \phi(t) d t\right) \\
& \leq \limsup _{n \rightarrow \infty} \alpha\left(G_{n-1}\right) \limsup \int_{n \rightarrow \infty}^{G_{n-1}} \phi(t) d t<\int_{0}^{c} \phi(t) d t .
\end{aligned}
$$

Which is contradiction. Hence

$\lim _{n \rightarrow \infty} G_{n}=0$.

Next, we prove that $\left\{y_{n}\right\}_{n \in \mathrm{N}}$ is a Cauchy sequence in $g(X)$. 
Suppose $\left\{y_{n}\right\}_{n \in \mathrm{N}}$ is not a Cauchy sequence. Then there exists $\varepsilon>0$ and for each positive integer $k$, there are positive integers $m(k)$ and $n(k)$ with $n(k)>m(k)>k$ such that

$G\left(y_{n(k)}, y_{m(k)}, y_{m(k)}\right) \geq \varepsilon$.

Corresponding to $m(k)$, we can choose $n(k)$ such that it is the smallest integer with $n(k)>m(k)$ and satisfying (3.6). Then

$G\left(y_{n(k)}, y_{m(k)-1}, y_{m(k)-1}\right)<\varepsilon$.

Then we have

$$
\begin{gathered}
\varepsilon \leq G\left(y_{n(k)}, y_{m(k)}, y_{m(k)}\right) \leq G\left(y_{n(k)}, y_{m(k)-1}, y_{m(k)-1}\right)+G\left(y_{m(k)-1}, y_{m(k)}, y_{m(k)}\right) . \\
<\varepsilon+G\left(y_{m(k)-1}, y_{m(k)}, y_{m(k)}\right) .
\end{gathered}
$$

Letting $k \rightarrow \infty$ and using (3.5) we get

$\lim _{k \rightarrow \infty} G\left(y_{m(k)}, y_{m(k)}, y_{n(k)}\right)=\varepsilon$.

Next by using Proposition 2.5 we can write

$$
\begin{aligned}
& \mid G\left(y_{m(k)}, y_{m(k)}, y_{n(k)}\right)-G\left(y_{m(k)}, y_{m(k)}, y_{n(k)+1} \mid\right. \\
& \leq \max \left\{G\left(y_{n(k)+1}, y_{n(k)}, y_{n(k)}\right), G\left(y_{n(k)}, y_{n(k)+1}, y_{n(k)+1}\right)\right\} \\
& \leq 2 G\left(y_{n(k)}, y_{n(k)+1}, y_{n(k)+2}\right)=2 G_{n}(k) ;
\end{aligned}
$$

$$
\begin{aligned}
& \mid G\left(y_{n(k)+1}, y_{m(k)+1}, y_{m(k)+1}\right)-G\left(y_{n(k)+1}, y_{m(k)+1}, y_{m(k)} \mid\right. \\
& \leq \max \left\{G\left(y_{m(k)}, y_{m(k)+1}, y_{m(k)+1}\right), G\left(y_{m(k)+1}, y_{m(k)}, y_{m(k)}\right\}\right. \\
& \leq 2 G\left(y_{m(k)}, y_{m(k)+1}, y_{m(k)+2}\right)=2 G_{m}(k) ;
\end{aligned}
$$

$$
\begin{aligned}
& \mid G\left(y_{m(k)+1}, y_{m(k)+1}, y_{n(k)+1}\right)-G\left(y_{m(k)+1}, y_{m(k)+1}, y_{n(k)+2} \mid\right. \\
& \leq \max \left\{G\left(y_{n(k)+2}, y_{n(k)+1}, y_{n(k)+1}\right), G\left(y_{n(k)+1}, y_{n(k)+2}, y_{n(k)+2}\right\}\right. \\
& \leq 2 G\left(y_{n(k)+1}, y_{n(k)+2}, y_{n(k)+3}\right)=2 G_{n(k)+1} .
\end{aligned}
$$

By letting $k \rightarrow \infty$ and taking into account (3.5), (3.8), (3.9), (3.10) and (3.11) we get the following system

$$
\left.\begin{array}{r}
\lim _{k \rightarrow \infty} G\left(y_{m(k)}, y_{m(k)}, y_{n(k)}\right)=\varepsilon, \\
\lim _{k \rightarrow \infty} G\left(y_{m(k)}, y_{m(k)}, y_{n(k)+1}\right)=\varepsilon, \\
\lim _{k \rightarrow \infty} G\left(y_{n(k)+1}, y_{m(k)+1}, y_{m(k)+1}\right)=\varepsilon, \\
\lim _{k \rightarrow \infty} G\left(y_{n(k)+1}, y_{m(k)+1}, y_{m(k)}\right)=\varepsilon, \\
\lim _{k \rightarrow \infty} G\left(y_{m(k)+1}, y_{m(k)+1}, y_{n(k)+2}\right)=\varepsilon .
\end{array}\right\}
$$

Taking limit superior and using (3.12) we have

$$
\begin{aligned}
0 & <\int_{0}^{\varepsilon} \phi(t) d t \\
& =\limsup _{k \rightarrow \infty} \int_{0}^{G\left(y_{m(k)+1}, y_{m(k)+1}, y_{n(k)+2}\right)} \phi(t) d t \\
& \leq \limsup _{k \rightarrow \infty} \alpha\left(G\left(y_{m(k)}, y_{m(k)}, y_{n(k)+1}\right) \int_{0}^{G\left(y_{m(k)}, y_{m(k)}, y_{n(k)+1}\right)} \phi(t) d t\right) \\
& \leq \limsup _{k \rightarrow \infty} \alpha\left(G\left(y_{m(k)}, y_{m(k)}, y_{n(k)+1}\right) \int_{0}^{\varepsilon} \phi(t) d t<\int_{0}^{\varepsilon} \phi(t) d t .\right.
\end{aligned}
$$

Again contradiction arises. Thus $\left\{y_{n}\right\}_{n \in \mathrm{N}}$ is a Cauchy sequence in $g(X)$ and since $g(X)$ is complete, there exists a point $r \in g(X)$ such that $\lim _{n \rightarrow \infty} y_{n}(x)=r$. So, there exist $w \in X$ such that $g w=r$. From Proposition 2.3 we have

$$
\lim _{n \rightarrow \infty} G\left(g x_{n}, g x_{n}, g w\right)=\lim _{n \rightarrow \infty} G\left(g x_{n}, g w, g w\right)=0 .
$$

Further we show that $g w=f w$. Suppose that $g w \neq f w$ then by using inequality, one can get

$$
\begin{aligned}
\int_{0}^{G\left(g x_{n}, f w, f w\right)} \phi(t) d t & =\int_{0}^{G\left(f x_{n-1}, f w, f w\right)} \phi(t) d t \\
& \leq \alpha\left(G\left(g x_{n-1}, g w, g w\right) \int_{0}^{G\left(g x_{n-1}, g w, g w\right)} \phi(t) d t\right.
\end{aligned}
$$

By taking $n \rightarrow \infty$ and using (3.14) we have,

$$
\int_{0}^{G(g w, f w, f w)} \phi(t) d t \leq 0,
$$

which contradict the fact that $g w \neq f w$. Thus $f w=g w$.

Uniqueness: We now show that $f$ and $g$ have a unique point of coincidence. Suppose that $f \xi=g \xi$ for some $\xi \in X$, the using inequality (3.1), we can write

$$
\begin{aligned}
\int_{0}^{G(g \xi, g w, g w)} \phi(t) d t & =\int_{0}^{G(f \xi, f w, f w)} \phi(t) d t \\
& \leq \alpha\left(G(g \xi, g w, g w) \int_{0}^{G(g \xi, g w, g w)} \phi(t) d t<\int_{0}^{G(g \xi, g w, g w)} \phi(t) d t\right.
\end{aligned}
$$

but this holds if $G(g \xi, g w, g w)=0$. Hence $g \xi=g w$. Which gives $f$ and $g$ have a unique point of coincident. From Proposition $2.4 f$ and $g$ have unique common fixed point.

Now we present some corollaries of Theorem 3.1.

Corollary 3.2. Let $\boldsymbol{\alpha}, G \boldsymbol{\complement}$ be complete G-metric space and $f, g: X \rightarrow X$ be a mapping such that

$$
\int_{0}^{G(f x, f y, f z)} \phi(t) d t \leq c \int_{0}^{G(g x, g y, g z)} \phi(t) d t
$$

for all $x, y, z \in X$ where $c \in[0,1)$ and $\phi \in \Phi$. If $f(X) \subset g(X)$ and $g(X)$ is a complete subspace of $X$. Then $f$ and $g$ have a unique point of coincidence in $X$. Moreover if $f$ and $g$ are weakly compatible, then $f$ and $g$ have a unique common fixed point in $X$.

Proof. It follows by taking $\alpha(s)=c$ in Theorem 3.1.

Corollary 3.3. Let $(X, G)$ be a complete G-metric space and $f, g: X \rightarrow X$ such that

$$
G(f x, f y, f z) \leq \alpha(G(g x, g y, g z)),
$$

for all $x, y, z \in X$ where $0 \geq \alpha<1$. If $f(X) \subset g(X)$ and $g(X)$ is a complete subspace of $X$, then $f$ and $g$ have a unique point of coincidence in $X$. Moreover if $f$ and $g$ are weakly compatible, then $f$ and $g$ have a unique common fixed point in $X$

Proof. It follows by taking $\phi(t)=1$ and $\alpha(x)=\alpha$ in Theorem 3.1. Corollary 3.4. Let $(X, G)$ be a complete G-metric space and $f: X \rightarrow X$ be a mapping such that

$$
\int_{0}^{G(f x, f y, f z)} \phi(t) d t \leq \alpha(G(x, y, z)) \int_{0}^{G(x, y, z)} \phi(t) d t,
$$

for all $x, y, z \in X$ where $\phi \in \Phi$ and $\alpha:[0,+\infty) \rightarrow[0,1)$ is a function with $\alpha(t)<1 \forall t \in \mathrm{R}^{+}, \limsup \alpha(s)<1, \forall t>0$

. Then $f$ has a unique fixed point in $X$.

Proof. The proof follows by taking $g(x)=x$ in Theorem 3.1.

Corollary 3.5. Let $(X, G)$ be a complete G-metric space and $f, g: X \rightarrow X$ such that

$$
G(f x, f y, f z) \leq \alpha(G(g x, g y, g z))(G(g x, g y, g z)),
$$
for all $x, y, z \in X$ where $\alpha:[0,+\infty) \rightarrow[0,1)$ is a function with $\alpha(t)<1 \forall t \in \mathrm{R}^{+}, \limsup \alpha(s)<1, \forall t>0$. If $f(X) \subset g(X)$ and $g(X)$ is a complete subspace of $X$, Then $f$ and $g$ have a unique point of coincidence in $X$. Moreover if $f$ and $g$ are weakly compatible, then $f$ and $g$ have a unique common fixed point in $X$.

Proof. It follows by taking $\phi(t)=1$ in Theorem 3.1.

Corollary 3.6. Let $(X, G)$ be complete G-metric space and $f: X \rightarrow X$ such that

$$
G(f x, f y, f z) \leq \alpha(G(x, y, z))(G(x, y, z)),
$$

for all $x, y, z \in X$ where $\alpha:[0,+\infty) \rightarrow[0,1)$ is a function with 
$\alpha(t)<1 \forall t \in \mathrm{R}^{+}, \limsup _{s \rightarrow t} \alpha(s)<1, \quad \forall t>0$. Then $f$ has a unique fixed point in $X$.

Proof. It follows by taking $g(x)=x$ and $\phi(t)=1$ in Theorem 3.1.

Corollary 3.7. Let $(X, G)$ be complete G-metric space and $f, g: X \rightarrow X$ such that

$\int_{0}^{G\left(f^{m} x, f^{m} y, f^{m} z\right)} \phi(t) d t \leq \alpha\left(G\left(g^{m} x, g^{m} y, g^{m} z\right)\right) \int_{0}^{\left(G\left(g^{m} x, g^{m} y, g^{m} z\right)\right)} \phi(t) d t$

for all $x, y, z \in X$ where $\phi \in \Phi$ and $\alpha:[0,+\infty) \rightarrow[0,1)$ is a function with $\quad \alpha(t)<1 \forall t \in \mathrm{R}^{+}, \quad \limsup _{s \rightarrow t} \alpha(s)<1, \quad \forall t>0$. If $f^{m}(X) \subset g^{m}(X)$ and $g(X)$ is a complete subspace of $X$, Then $f$ and $g$ have a unique point of coincidence in $X$. Moreover, if $f^{m}$ and $g^{m}$ are weakly compatible, then $f$ and $g$ have a unique common fixed point $\alpha \in X$.

Proof. It is follow from Theorem 3.1 that $f^{m}(x), g^{m}(x)$ have a unique common fixed point $\quad$ Now $f(\alpha)=f\left(f^{m}(\alpha)\right)=f^{m+1}(\alpha)=f^{m}(f(\alpha))$, $g(\alpha)=g\left(g^{m}(\alpha)\right)=g^{m+1}(\alpha)=g^{m}(g(\alpha))$ implies that $f \alpha$, $g \alpha$ are also fixed point for $f^{m}(x), g^{m}(x)$. Since the common fixed point of $f^{m}(x), g^{m}(x)$ is unique. Hence, $\alpha=f \alpha=g \alpha$.

Theorem 3.8. Let $(X, G)$ be complete G-metric space and $f, g: X \rightarrow X$ be a mapping such that for all $x, y, z \in X$

$$
\int_{0}^{G(f x, f y, f z)} \phi(t) d t \leq \alpha(G(g x, g y, g z)) \int_{0}^{(G(g x, f x, g x))} \phi(t) d t
$$

$+\beta(G(g x, g y, g z)) \int_{0}^{(G(g y, f y, g x))} \phi(t) d t+\gamma(G(g x, g y, g z)) \int_{0}^{(G(g x, g y, g z))} \phi(t) d t$,

where $\phi \in \Phi$ and $\alpha, \beta, \gamma:[0,+\infty) \rightarrow[0,1)$ are functions such that

$\alpha(t)+\beta(t)+\gamma(t)<1 \forall t \in \mathrm{R}^{+}$,

and

$\limsup [\alpha(t)+\beta(t)+\gamma(t)]<1 \forall t>0$.

If $f(X) \subset g(X)$ and $g(X)$ is a complete subspace of $X$. Then $f$ and $g$ have a unique point of coincidence in $X$. Moreover if $f$ and $g$ are weakly compatible, then $f$ and $g$ have a unique common fixed point in $X$.

Proof. Let $x_{0}$ be arbitrary point in $X$. Since $f(X) \subset g(X)$ choose $x_{1} \in X$ such that $g x_{1}=f x_{0}$ choose $x_{2} \in X$ such that $g x_{2}=f x_{1}$ in last we choose $x_{n+1} \in X$ such that $y_{n}=g x_{n+1}=f x_{n}$. Let $G_{n}=G\left(y_{n}, y_{n+1}, y_{n+2}\right)$ by using (3.15)

$$
\begin{aligned}
\int_{0}^{G_{n}} \phi(t) d t & =\int_{0}^{G\left(f x_{n}, f x_{n+1}, f x_{n+2}\right)} \phi(t) d t \\
& \leq \alpha\left(G\left(g x_{n}, g x_{n+1}, g x_{n+2}\right)\right) \int_{0}^{G\left(g x_{n}, f x_{n}, g x_{n}\right)} \phi(t) d t \\
& +\beta\left(G\left(g x_{n}, g x_{n+1}, g x_{n+2}\right)\right) \int_{0}^{G\left(g x_{n+1}, f x_{n+1}, g x_{n}\right)} \phi(t) d t \\
& +\gamma\left(G\left(g x_{n}, g x_{n+1}, g x_{n+2}\right)\right) \int_{0}^{G\left(g x_{n}, g x_{n+1}, g x_{n+2}\right)} \phi(t) d t
\end{aligned}
$$

Using symmetric property of $G$-metric and $G(x, x, y) \leq G(x, y, z)$ we get
$\int_{0}^{G_{n}} \phi(t) d t \leq \alpha\left(G_{n-1}\right) \int_{0}^{G_{n-1}} \phi(t) d t+\beta\left(G_{n-1}\right) \int_{0}^{G_{n-1}} \phi(t) d t+$

$$
\begin{aligned}
\gamma\left(G_{n-1}\right) \int_{0}^{G_{n-1}} \phi(t) d t & =\left(\alpha\left(G_{n-1}\right)+\gamma\left(G_{n-1}\right)+\beta\left(G_{n-1}\right)\right) \int_{0}^{G_{n-1}} \phi(t) d t \\
& \leq \int_{0}^{G_{n-1}} \phi(t) d t, \quad \forall n \in \mathrm{N} .
\end{aligned}
$$

Thus

$$
G_{n} \leq G_{n-1}, \forall n \in \mathrm{N} \text {. }
$$

Which shows that the sequence $\left\{G_{n}\right\}_{n \in \mathrm{N}}$ is decreasing. Consequently, them exist a constant $C$ such that

$$
\lim _{n \rightarrow \infty} G_{n}=c
$$

We show that $c=0$, Otherwise if $c>0$ then from above we have

$$
\int_{0}^{G_{n}} \phi(t) d t \leq\left(\alpha\left(G_{n-1}\right)+\beta\left(G_{n-1}\right)+\gamma\left(G_{n-1}\right)\right) \int_{0}^{G_{n-1}} \phi(t) d t
$$

Taking limit superior and using (3.17) and (3.19) we get

$$
\begin{aligned}
0<\int_{0}^{c} \phi(t) d t & =\limsup _{n \rightarrow \infty} \int_{0}^{G_{n}} \phi(t) d t \\
& \leq \limsup _{n \rightarrow \infty}\left(\alpha\left(G_{n-1}\right)+\beta\left(G_{n-1}\right)+\gamma\left(G_{n-1}\right)\right) \limsup _{n \rightarrow \infty} \int_{0}^{G_{n-1}} \phi(t) d t \\
& <\int_{0}^{c} \phi(t) d t .
\end{aligned}
$$

Which is contradiction. Hence

$$
\lim _{n \rightarrow \infty} G_{n}=0
$$

Next, we prove that $\left\{y_{n}\right\}_{n \in \mathrm{N}}$ is a Cauchy sequence in $g(X)$. Suppose $\left\{y_{n}\right\}_{n \in \mathrm{N}}$ is not a Cauchy sequence. Then there exists $\varepsilon>0$ and for each positive integer $k$, there are positive integers $m(k)$ and $n(k)$ with $n(k)>m(k)>k$ such that

$$
G\left(y_{n(k)}, y_{m(k)}, y_{m(k)}\right) \geq \varepsilon \text {. }
$$

Corresponding to $m(k)$, we can choose $n(k)$ such that it is the smallest integer with $n(k)>m(k)$ and satisfying (3.21). Then

$$
G\left(y_{n(k)}, y_{m(k)-1}, y_{m(k)-1}\right)<\varepsilon .
$$

Then we have

$$
\begin{gathered}
\varepsilon \leq G\left(y_{n(k)}, y_{m(k)}, y_{m(k)}\right) \leq G\left(y_{n(k)}, y_{m(k)-1}, y_{m(k)-1}\right)+G\left(y_{m(k)-1}, y_{m(k)}, y_{m(k)}\right) . \\
<\varepsilon+G\left(y_{m(k)-1}, y_{m(k)}, y_{m(k)}\right) .
\end{gathered}
$$

Setting $k \rightarrow \infty$ and using (3.21) we have,

$$
\lim _{k \rightarrow \infty} G\left(y_{m(k)}, y_{m(k)}, y_{n(k)}\right)=\varepsilon \text {. }
$$

Now by using Proposition 2.5 we get the next three inequalities

$$
\begin{aligned}
& \mid G\left(y_{m(k)}, y_{m(k)}, y_{n(k)}\right)-G\left(y_{m(k)}, y_{m(k)}, y_{n(k)+1} \mid\right. \\
& \leq \max \left\{G\left(y_{n(k)+1}, y_{n(k)}, y_{n(k)}\right), G\left(y_{n(k)}, y_{n(k)+1}, y_{n(k)+1}\right\}\right. \\
& \leq 2 G\left(y_{n(k)}, y_{n(k)+1}, y_{n(k)+2}\right)=2 G_{n}(k) ;
\end{aligned}
$$

$$
\begin{aligned}
& \mid G\left(y_{n(k)+1}, y_{m(k)+1}, y_{m(k)+1}\right)-G\left(y_{n(k)+1}, y_{m(k)+1}, y_{m(k)} \mid\right. \\
& \leq \max \left\{G\left(y_{m(k)+1}, y_{m(k)}, y_{m(k)}\right), G\left(y_{m(k)}, y_{m(k)+1}, y_{m(k)+1}\right\}\right. \\
& \leq 2 G\left(y_{m(k)}, y_{m(k)+1}, y_{m(k)+2}\right)=2 G_{m}(k) ;
\end{aligned}
$$


And

$$
\begin{aligned}
& \mid G\left(y_{m(k)+1}, y_{m(k)+1}, y_{n(k)+1}\right)-G\left(y_{m(k)+1}, y_{m(k)+1}, y_{n(k)+2} \mid\right. \\
& \leq \max \left\{G\left(y_{n(k)+1}, y_{n(k)+1}, y_{n(k)+2}\right), G\left(y_{n(k)+2}, y_{n(k)+1}, y_{n(k)+1}\right\}\right. \\
& \leq 2 G\left(y_{n(k)+1}, y_{n(k)+1}, y_{n(k)+3}\right)=2 G_{n(k)+1} .
\end{aligned}
$$

Letting $k \rightarrow \infty$ and combining (3.20), (3.23), (3.24), (3.25) and (3.26) one can get

$$
\left.\begin{array}{r}
\lim _{k \rightarrow \infty} G\left(y_{m(k)}, y_{m(k)}, y_{n(k)}\right)=\varepsilon, \\
\lim _{k \rightarrow \infty} G\left(y_{m(k)}, y_{m(k)}, y_{n(k)+1}\right)=\varepsilon, \\
\lim _{k \rightarrow \infty} G\left(y_{n(k)+1}, y_{m(k)+1}, y_{m(k)+1}\right)=\varepsilon, \\
\lim _{k \rightarrow \infty} G\left(y_{n(k)+1}, y_{m(k)+1}, y_{m(k)}\right)=\varepsilon, \\
\lim _{k \rightarrow \infty} G\left(y_{m(k)+1}, y_{m(k)+1}, y_{n(k)+2}\right)=\varepsilon .
\end{array}\right\}
$$

Using (3.15) we can write

$$
\begin{gathered}
\int_{0}^{G\left(y_{m(k)+1}, y_{m(k)+1}, y_{n(k)+2)}\right.} \phi(t) d t \\
\leq \alpha\left(G\left(y_{m(k)}, y_{m(k)}, y_{n(k)+1}\right) \int_{0}^{G\left(y_{m(k)}, y_{m(k)+1}, y_{m(k)}\right)} \phi(t) d t\right. \\
+\beta\left(G\left(y_{m(k)}, y_{m(k)}, y_{n(k)+1}\right) \int_{0}^{G\left(y_{m(k)}, y_{m(k)+1}, y_{m(k)}\right)} \phi(t) d t\right. \\
+\gamma\left(G\left(y_{m(k)}, y_{m(k)}, y_{n(k)+1}\right) \int_{0}^{G\left(y_{m(k)}, y_{m(k)}, y_{n(k)+1}\right)} \phi(t) d t, \forall k \in \mathrm{N} .\right.
\end{gathered}
$$

Also

$$
\begin{aligned}
G\left(y_{m(k)}, y_{m(k)+1}, y_{m(k)}\right) & =G\left(y_{m(k)}, y_{m(k)}, y_{m(k)+1}\right) \\
& \leq G\left(y_{m(k)}, y_{m(k)+1}, y_{m(k)+2}\right) .
\end{aligned}
$$

Taking limit superior of (3.28) and using (3.20), (3.27) and (3.29) we get

$$
\begin{gathered}
0<\int_{0}^{\varepsilon} \phi(t) d t=\limsup _{k \rightarrow \infty} \int_{0}^{G\left(y_{m(k)+1}, y_{m(k)+1}, y_{n(k)+2}\right)} \phi(t) d t \\
\left.\leq \limsup _{k \rightarrow \infty} \gamma\left(G\left(y_{m(k)}, y_{m(k)}, y_{n(k)+1}\right)\right) \int_{0}^{G\left(y_{m(k)}, y_{m(k)}, y_{n(k)+1}\right)} \phi(t) d t\right) \\
\leq \limsup _{k \rightarrow \infty} \gamma\left(G\left(y_{m(k)}, y_{m(k)}, y_{n(k)+1}\right)\right) \limsup _{k \rightarrow \infty} \int_{0}^{G\left(y_{m(k)}, y_{m(k)}, y_{n(k+1)}\right)} \phi(t) d t \\
\leq \limsup _{k \rightarrow \infty}\left(\gamma \left(G\left(y_{m(k)}, y_{m(k)}, y_{n(k)+1}\right) \int_{0}^{\varepsilon} \phi(t) d t<\int_{0}^{\varepsilon} \phi(t) d t\right.\right.
\end{gathered}
$$

which is contradiction. Thus $\left\{y_{n}\right\}_{n \in \mathrm{N}}$ is a Cauchy sequence in $g(X)$ and since $g(X)$ is complete, there exists a point $r \in g(X)$ such that $\lim _{n \rightarrow \infty} y_{n}(x)=r$. So, there exist $w \in X$ such that $g w=r$. From Proposition 2.3 we have

$$
\begin{aligned}
\lim _{n \rightarrow \infty} G\left(g x_{n}, g x_{n}, g w\right) & =\lim _{n \rightarrow \infty} G\left(g x_{n}, g w, g w\right)=0 . \\
(3.30) &
\end{aligned}
$$

Now we will show that $g w=f w$. Suppose that $g w \neq f w$ by (3.15), we have

$$
\begin{aligned}
\int_{0}^{G\left(g x_{n}, f w, g w\right)} \phi(t) d t & =\int_{0}^{G\left(f x_{n-1}, f w, f w\right)} \phi(t) d t \\
& \leq \alpha\left(G\left(g x_{n-1}, g w, g w\right) \int_{0}^{G\left(g x_{n-1}, f x_{n-1}, g x_{n-1}\right)} \phi(t) d t\right. \\
& +\beta\left(G\left(g x_{n-1}, g w, g w\right)\right) \int_{0}^{G\left(g w, f w, g x_{n-1}\right)} \phi(t) d t \\
& +\gamma\left(G\left(g x_{n-1}, g w, g w\right) \int_{0}^{G\left(g x_{n-1}, g w, g w\right)} \phi(t) d t\right.
\end{aligned}
$$

By taking $n \rightarrow \infty$ and using (3.30) we get

$$
\int_{0}^{G(g w, f w, g w)} \phi(t) d t \leq \beta\left(G\left(g x_{n-1}, g w, g w\right) \int_{0}^{G(g w, f w, g w)} \phi(t) d t\right.
$$

which contradict the fact that $f w \neq g w$. Hence $g w=f w$.

Uniqueness: By following the lines in the proof of Theorem 3.1 we conclude the uniqueness of fixed point.

Theorem 3.8 yields the following corollaries:

Corollary 3.9. Let $(X, G)$ be complete G-metric space and $f, g: X \rightarrow X$ be a mapping such that for all $x, y, z \in X$ $\int_{0}^{G(f x, f y, f z)} \phi(t) d t \leq \alpha(G(g x, g y, g z))\left(\int_{0}^{(G(g x, f x, g x))} \phi(t) d t+\int_{0}^{(G(g y, f y, g x))} \phi(t) d t\right.$

$$
\left.+\int_{0}^{(G(g x, g y, g z))} \phi(t) d t\right)
$$

where $\phi \in \Phi$ and $\alpha:[0,+\infty) \rightarrow[0,1)$ is a function with $\alpha(t)<1$ for all $t \in \mathrm{R}^{+}$and $\limsup _{s \rightarrow t}[\alpha(s)]<1$ for all $t>0$. If $f(X) \subset g(X)$ and $g(X)$ is a complete subspace of $X$. Then $f$ and $g$ have a unique point of coincidence in $X$. Moreover if $f$ and $g$ are weakly compatible, then $f$ and $g$ have a unique common fixed point in $X$.

Proof. It follows by taking $\alpha(s)=\beta(s)=\gamma(s)$ in Theorem 3.8.

Corollary 3.10. Let $(X, G)$ be complete G-metric space and $f, g: X \rightarrow X$ be a mapping such that for all $x, y, z \in X$.

$$
\begin{aligned}
\int_{0}^{G(f x, f y, f z)} \phi(t) d t & \leq a \int_{0}^{(G(g x, f x, g x))} \phi(t) d t+b \int_{0}^{(G(g y, f y, g x))} \phi(t) d t \\
& +c \int_{0}^{(G(g x, g y, g z))} \phi(t) d t,
\end{aligned}
$$

where $\phi \in \Phi$ and $a+b+c<1$. If $f(X) \subset g(X)$ and $g(X)$ is a complete subspace of $X$. Then $f$ and $g$ have a unique point of coincidence in $X$. Moreover if $f$ and $g$ are weakly compatible, then $f$ and $g$ have a unique common fixed point in $X$.

Proof. It follows by taking $\alpha(s)=a, \beta(s)=b, \gamma(s)=c$ in Theorem 3.8.

Corollary 3.11. Let $(X, G)$ be complete $\mathrm{G}$-metric space and $f, g: X \rightarrow X$ be a mapping such that for all $x, y, z \in X$

$$
\begin{gathered}
\int_{0}^{G(f x, f y, f z)} \phi(t) d t \leq \alpha(G(x, y, z)) \int_{0}^{(G(x, f x, x))} \phi(t) d t \\
+\beta(G(x, y, z)) \int_{0}^{(G(y, f y, x))} \phi(t) d t+\gamma(G(x, y, z)) \int_{0}^{(G(x, y, z))} \phi(t) d t,
\end{gathered}
$$

where $\phi \in \Phi$ and $\alpha, \beta, \gamma:[0,+\infty) \rightarrow[0,1)$ are functions such that $\quad \alpha(t)+\beta(t)+\gamma(t)<1, \forall t \in \mathrm{R}^{+} \quad$ and $\limsup [\alpha(s)+\beta(s)+\gamma(s)]<1, \forall t>0$. If $\quad f(X) \subset g(X) \quad$ and $g(X)$ is a complete subspace of $X$. Then $f$ and $g$ have a unique point of coincidence in $X$. Moreover if $f$ and $g$ are weakly compatible, then $f$ and $g$ have a unique common fixed point in $X$.

Proof. The proof follows by taking $g(x)=x$ in Theorem 3.8.

Corollary 3.12. Let $(X, G)$ be complete G-metric space and $f, g: X \rightarrow X$ be a mapping such that for all $x, y, z \in X$

$$
\begin{aligned}
\int_{0}^{G\left(f^{m} x, f^{m} y, f^{m} z\right)} \phi(t) d t & \leq \alpha\left(G\left(g^{m} x, g^{m} y, g^{m} z\right)\right) \int_{0}^{\left(G\left(g^{m} x, f^{m} x, g^{m} x\right)\right)} \phi(t) d t \\
& +\beta\left(G\left(g^{m} x, g^{m} y, g^{m} z\right)\right) \int_{0}^{\left(G\left(g^{m} y, f^{m} y, g^{m} x\right)\right)} \phi(t) d t \\
& +\gamma\left(G\left(g^{m} x, g^{m} y, g^{m} z\right)\right) \int_{0}^{\left(G\left(g^{m} x, g^{m} y, g^{m} z\right)\right)} \phi(t) d t,
\end{aligned}
$$

where $\phi \in \Phi$ and $\alpha, \beta, \gamma:[0,+\infty) \rightarrow[0,1)$ are functions such that $\quad \alpha(t)+\beta(t)+\gamma(t)<1, \forall t \in \mathrm{R}^{+} \quad$ and $\limsup _{s \rightarrow t}[\alpha(s)+\beta(s)+\gamma(s)]<1, \forall t>0$. If $f^{m}(X) \subset g^{m}(X)$ and 
$g(X)$ is a complete subspace of $X$. Then $f$ and $g$ have a unique point of coincidence in $X$. Moreover if $f$ and $g$ are weakly compatible, then $f$ and $g$ have a unique common fixed point in $X$.

Proof. The proof is same as of the Corollary 3.7 .

Corollary 3.13. Let $(X, G)$ be complete G-metric space and $f, g: X \rightarrow X$ be a mapping such that for all $x, y, z \in X$

$$
G(f x, f y, f z) \leq \alpha(G(g x, g y, g z))(G(g x, f x, g x))
$$

$+\beta(G(g x, g y, g z))(G(g y, f y, g x))+\gamma(G(g x, g y, g z))(G(g x, g y, g z))$,

where $\quad \alpha, \beta, \gamma:[0,+\infty) \rightarrow[0,1)$ are function such that $\alpha(t)+\beta(t)+\gamma(t)<1, \forall t \in \mathrm{R}^{+} \quad$ and $\limsup [\alpha(s)+\beta(s)+\gamma(s)]<1, \forall t>0$. If $f(X) \subset g(X)$ and $g(X)$ is a complete subspace of $X$. Then $f$ and $g$ have a unique point of coincidence in $X$. Moreover if $f$ and $g$ are weakly compatible, then $f$ and $g$ have a unique common fixed point in $X$.

Proof. The proof follows by taking $\phi(t)=1$ in Theorem 3.8.

Corollary 3.14. Let $(X, G)$ be complete G-metric space and $f, g: X \rightarrow X$ be a mapping such that for all $x, y, z \in X$

$G(f x, f y, f z) \leq a(G(g x, f x, g x))+b(G(g y, f y, g x))+c(G(g x, g y, g z))$, where $a+b+c<1$. If $f(X) \subset g(X)$ and $g(X)$ is a complete subspace of $X$. Then $f$ and $g$ have a unique point of coincidence in $X$. Moreover, if $f$ and $g$ are weakly compatible, then $f$ and $g$ have a unique common fixed point in $X$.

Proof. The proof follows by taking $\phi(t)=1$ and $\alpha(s)=a, \beta(s)=b, \gamma(s)=c$ in Theorem 3.8.

Similar to Theorem 3.8 we can prove the following theorem. Theorem 3.15. Let $(X, G)$ be complete G-metric space and $f, g: X \rightarrow X$ be a mapping such that for all $x, y, z \in X$

$$
\begin{aligned}
\int_{0}^{G(f x, f y, f z)} \phi(t) d t & \leq \alpha(G(g x, g y, g z)) \int_{0}^{(G(g x, f x, g x))} \phi(t) d t \\
& +\beta(G(g x, g y, g z)) \int_{0}^{(G(g y, f y, g x))} \phi(t) d t,
\end{aligned}
$$

where $\phi \in \Phi$ and $\alpha, \beta:[0,+\infty) \rightarrow[0,1)$ is a function with $\alpha(t)+\beta(t)<1, \forall t \in \mathrm{R}^{+} \quad$ and $\quad \limsup [\alpha(s)+\beta(s)]<1, \forall t>0 . \quad$ If $f(X) \subset g(X)$ and $g(X)$ is a complete subspace of $X$. Then $f$ and $g$ have a unique point of coincidence in $X$. Moreover if $f$ and $g$ are weakly compatible, then $f$ and $g$ have a unique common fixed point in $X$.

Example 3.16. Let $X=[0,1]$ and defined $G: X \times X \times X \rightarrow \mathrm{R}^{+}$by $G(x, y, z)=|x-y|+|y-z|+|z-x| \forall x, y, z \in X$.

Then $(X, G)$ is complete G-metric space. Let $f, g: X \rightarrow X$, $\phi: \mathrm{R}^{+} \rightarrow \mathrm{R}^{+}$and $\alpha: \mathrm{R}^{+} \rightarrow[0,1)$ define by

$$
\begin{gathered}
f(x)=\frac{x}{9}, \quad \forall x \in[0,1] ; \\
g(x)=\frac{x}{3}, \quad \forall x \in[0,1] ; \\
\phi(t)=2 t, \quad \forall t \in[0, \infty) ; \\
\alpha(t)=\frac{1}{(t+3)}, \quad \forall t \in[0, \infty) .
\end{gathered}
$$

Clearly $f(X) \subset g(X)$ also $f$ and $g$ are weakly compatible. Now we have

$$
\begin{gathered}
\int_{0}^{G(f x, f y, f z)} \phi(t) d t=(G(f x, f y, f z))^{2}=\frac{(G(x, y, z))^{2}}{81} \\
\leq \frac{(G(x, y, z))^{2}}{9((G(x, y, z))+3)}=\alpha(G(g x, g y, g z)) \int_{0}^{G(g x, g y, g z)} \phi(t) d t .
\end{gathered}
$$

Thus all conditions of Theorem 3.1 are satisfied. Hence $f$ and $g$ have a unique common fixed point which is 0 .

The following example shows that Theorem 3.1 is more general than Theorem 14.

Example 3.17. Let $X=\left[0, \frac{1}{2}\right]$ and defined $G: X \times X \times X \rightarrow \mathrm{R}^{+}$ by

$G(x, y, z)=|x-y|+|y-z|+|z-x| \forall x, y, z \in X$.

Then $(X, G)$ is complete G-metric space. Let $f, g: X \rightarrow X$,

$\phi: \mathrm{R}^{+} \rightarrow \mathrm{R}^{+}$and $\alpha: \mathrm{R}^{+} \rightarrow[0,1)$ defined by

$$
\begin{gathered}
f(x)= \begin{cases}\frac{1}{4}, & \text { if } 0 \leq x \leq \frac{1}{4}, \\
0, & \text { if } \frac{1}{4}<x \leq \frac{1}{2}\end{cases} \\
g(x)= \begin{cases}x, & \text { if } 0 \leq x \leq \frac{1}{4}, \\
\frac{1}{2}, & \text { if } \frac{1}{4}<x \leq \frac{1}{2}\end{cases} \\
\phi(t)= \begin{cases}e^{-t}, & \text { if } t>1, \\
0, & \text { otherwise. }\end{cases} \\
\alpha(t)=\frac{1}{2(t+4)}, t \in[0,+\infty) .
\end{gathered}
$$

Clearly $f(X) \subset g(X)$ also $f$ and $g$ are weakly compatible. Now for all $x, y, z \in X \quad G(f x, f y, f z) \leq \frac{3}{4} \quad$ and hence $\int_{0}^{G(f x, f y, f z)} \phi(t) d t=0$.

Clearly, all conditions of Theorem 3.1 are satisfied. So $f$ and $g$ have a unique common fixed point.

On the other hand, if we take $x=\frac{1}{4}$ and $y=z=\frac{1}{2}$, then evidently

$$
\begin{aligned}
& G\left(f\left(\frac{1}{4}\right), f\left(\frac{1}{2}\right), f\left(\frac{1}{2}\right)\right)=\left|f\left(\frac{1}{4}\right)-f\left(\frac{1}{2}\right)\right|+\left|f\left(\frac{1}{2}\right)-f\left(\frac{1}{2}\right)\right|+\left|f\left(\frac{1}{2}\right)-f\left(\frac{1}{4}\right)\right|=\frac{1}{2}, \\
& \text { and } \\
& G\left(g\left(\frac{1}{4}\right), g\left(\frac{1}{2}\right), g\left(\frac{1}{2}\right)\right)=\left|g\left(\frac{1}{4}\right)-g\left(\frac{1}{2}\right)\right|+\left|g\left(\frac{1}{2}\right)-g\left(\frac{1}{2}\right)\right|+\left|g\left(\frac{1}{2}\right)-g\left(\frac{1}{4}\right)\right|=\frac{1}{2} .
\end{aligned}
$$

So we have the following bound

$$
G(f x, f y, f z)>k(G(g x, g y, g z)) .
$$

From the inequality, it is clear that Theorem th1.51 fail to guaranty that $f$ and $g$ have a unique common fixed point.

Example 3.18. Let $X=[0,1]$ and defined $G: X \times X \times X \rightarrow \mathrm{R}^{+}$by

$G(x, y, z)=\max \{|x-y|,|y-z|,|z-x|\}, \forall x, y, z \in X$. Then $(X, G)$ is complete G-metric space. Let $f, g: X \rightarrow X$, $\phi: \mathrm{R}^{+} \rightarrow \mathrm{R}^{+}$and $\alpha, \beta, \gamma:[0,+\infty) \rightarrow[0,1)$ defined by

$$
\begin{gathered}
f(x)= \begin{cases}\frac{\sqrt{x}}{12}, & \text { if } 0 \leq x<\frac{1}{2}, \\
\frac{\sqrt{x}}{6}, & \text { if } \frac{1}{2} \leq x \leq 1 .\end{cases} \\
g(x)= \begin{cases}\frac{\sqrt{x}}{4}, & \text { if } 0 \leq x<\frac{1}{2}, \\
\frac{\sqrt{x}}{2}, & \text { if } \frac{1}{2} \leq x \leq 1 .\end{cases} \\
\phi(t)=3 t^{2}, \quad \forall t \in[0, \infty), \\
\alpha(t)=\beta(t)=\gamma(t)=\frac{1}{\left(t^{2}+2\right)^{2}}, \forall t \in \mathrm{R}^{+},
\end{gathered}
$$

clearly $f(X) \subset g(X)$ also $f$ and $g$ are weakly compatible and 
$g(X)$ are complete subspace. Without loss of generality, we suppose that $x \leq y \leq z$ for all $x, y, z \in X$. To check the validity of (3.15) we distinguish the following cases:

Case 1. If $x, y, z \in\left[0, \frac{1}{2}\right)$, then

$\int_{0}^{G(f x, f y, f z)} \phi(t) d t=(G(f x, f y, f z))^{3}=(\max \{|f x-f y|,|f y-f z|,|f z-f x|\})^{3}$

$$
\begin{gathered}
=\left|\frac{\sqrt{z}}{12}-\frac{\sqrt{x}}{12}\right|^{3}=\frac{1}{27}\left|\frac{\sqrt{z}}{4}-\frac{\sqrt{x}}{4}\right|^{3} \\
\leq \frac{1}{27}\left(\left|\frac{\sqrt{x}}{6}\right|^{3}+\left|\frac{\sqrt{y}}{6}\right|^{3}+\left|\frac{\sqrt{z}}{4}-\frac{\sqrt{x}}{4}\right|^{3}\right) \\
\leq \frac{1024}{4225}\left(\left|\frac{\sqrt{x}}{6}\right|^{3}+\left|\frac{\sqrt{y}}{6}\right|^{3}+\left|\frac{\sqrt{z}}{4}-\frac{\sqrt{x}}{4}\right|^{3}\right) \\
\leq \frac{1}{\left.\left(\left|\frac{\sqrt{z}}{4}-\frac{\sqrt{x}}{4}\right|^{2}\right)+2\right)^{2}}\left(\left|\frac{\sqrt{x}}{6}\right|^{3}+\left|\frac{\sqrt{y}}{6}\right|^{3}+\left|\frac{\sqrt{z}}{4}-\frac{\sqrt{x}}{4}\right|^{3}\right) \\
=\alpha(G(g x, g y, g z)) \int_{0}^{(G(g x, f x, g x))} \phi(t) d t+\beta(G(g x, g y, g z)) \int_{0}^{(G(g y, f y, g x))} \phi(t) d t \\
+\gamma(G(g x, g y, g z)) \int_{0}^{(G(g x, g y, g z))} \phi(t) d t .
\end{gathered}
$$

Case 2. If $x, y, z \in\left[\frac{1}{2}, 1\right]$, then we can write

$$
\begin{aligned}
& \int_{0}^{G(f(x, y y, f z)} \phi(t) d t=(G(f x, f y, f z))^{3}=(\max \{|f x-f y|,|f y-f z||| f z-f x \mid\})^{3} \\
&=\left|\frac{\sqrt{z}}{6}-\frac{\sqrt{x}}{6}\right|^{3}=\frac{1}{27}\left|\frac{\sqrt{z}}{2}-\frac{\sqrt{x}}{2}\right|^{3} \\
& \leq \frac{1}{27}\left(\left|\frac{\sqrt{x}}{3}\right|^{3}+\left|\frac{\sqrt{y}}{3}\right|^{3}+\left|\frac{\sqrt{z}}{2}-\frac{\sqrt{x}}{2}\right|^{3}\right) \\
& \leq \frac{4}{25}\left(\left|\frac{\sqrt{x}}{3}\right|^{3}+\left|\frac{\sqrt{y}}{3}\right|^{3}+\left|\frac{\sqrt{z}}{2}-\frac{\sqrt{x}}{2}\right|^{3}\right) \\
& \leq \frac{1}{\left.\left(\left|\frac{\sqrt{z}}{2}-\frac{\sqrt{x}}{2}\right|^{2}\right)+2\right)^{2}}\left(\left|\frac{\sqrt{x}}{3}\right|^{3}+\left|\frac{\sqrt{y}}{3}\right|^{3}+\left|\frac{\sqrt{z}}{2}-\frac{\sqrt{x}}{2}\right|^{3}\right) \\
&=\alpha(G(g x, g y, g z)) \int_{0}^{(G(g x, f x, g x))} \phi(t) d t+\beta(G(g x, g y, g z)) \int_{0}^{(G(g y, f y, g x))} \phi(t) d t \\
&+\gamma(G(g x, g y, g z)) \int_{0}^{(G(g x, g y, g z))} \phi(t) d t .
\end{aligned}
$$

Case 3. If $x \in\left[0, \frac{1}{2}\right)$ and $y, z \in\left[\frac{1}{2}, 1\right]$, then

$$
\begin{gathered}
g(x)= \begin{cases}\frac{\sqrt{x}}{4}, & \text { if } 0 \leq x<\frac{1}{2}, \\
\frac{\sqrt{x}}{2}, & \text { if } \frac{1}{2} \leq x \leq 1 .\end{cases} \\
\phi(t)=3 t^{2}, \quad \forall t \in[0, \infty), \\
\alpha(t)=\beta(t)=\gamma(t)=\frac{1}{\left(t^{2}+2\right)^{2}}, \forall t \in \mathrm{R}^{+},
\end{gathered}
$$

clearly $f(X) \subset g(X)$ also $f$ and $g$ are weakly compatible and $g(X)$ are complete subspace. Without loss of generality, we suppose that $x \leq y \leq z$ for all $x, y, z \in X$. To check the validity of (3.15) we distinguish the following cases:

Case 1. If $x, y, z \in\left[0, \frac{1}{2}\right)$, then

$$
\begin{gathered}
\int_{0}^{G(f x, f y, f z)} \phi(t) d t=(G(f x, f y, f z))^{3}=(\max \{|f x-f y|,|f y-f z|,|f z-f x|\})^{3} \\
=\left|\frac{\sqrt{z}}{12}-\frac{\sqrt{x}}{12}\right|^{3}=\frac{1}{27}\left|\frac{\sqrt{z}}{4}-\frac{\sqrt{x}}{4}\right|^{\beta} \\
\leq \frac{1}{27}\left(\left|\frac{\sqrt{x}}{6}\right|^{\beta}+\left|\frac{\sqrt{y}}{6}\right|^{3}+\left|\frac{\sqrt{z}}{4}-\frac{\sqrt{x}}{4}\right|^{3}\right) \\
\leq \frac{1024}{4225}\left(\left|\frac{\sqrt{x}}{6}\right|^{\beta}+\left|\frac{\sqrt{y}}{6}\right|^{\beta}+\left|\frac{\sqrt{z}}{4}-\frac{\sqrt{x}}{4}\right|^{\beta}\right) \\
\leq \frac{1}{\left.\left(\left|\frac{\sqrt{z}}{4}-\frac{\sqrt{x}}{4}\right|^{2}\right)+2\right)^{2}}\left(\left|\frac{\sqrt{x}}{6}\right|^{\beta}+\left|\frac{\sqrt{y}}{6}\right|^{\beta}+\left|\frac{\sqrt{z}}{4}-\frac{\sqrt{x}}{4}\right|^{\beta}\right) \\
=\alpha(G(g x, g y, g z)) \int_{0}^{(G(g x, f x, g x))} \phi(t) d t+\beta(G(g x, g y, g z)) \int_{0}^{(G(g y, f y, g x))} \phi(t) d t \\
+\gamma(G(g x, g y, g z)) \int_{0}^{(G(g x, g y, g z))} \phi(t) d t .
\end{gathered}
$$

Case 2. If $x, y, z \in\left[\frac{1}{2}, 1\right]$, then we can write
$\int_{0}^{G(f x, f y, f z)} \phi(t) d t=(G(f x, f y, f z))^{3}=(\max \{|f x-f y|,|f y-f z|,|f z-f x|\})^{3}$

$$
\begin{gathered}
=\left|\frac{\sqrt{z}}{6}-\frac{\sqrt{x}}{6}\right|^{3}=\frac{1}{27}\left|\frac{\sqrt{z}}{2}-\frac{\sqrt{x}}{2}\right|^{3} \\
\leq \frac{1}{27}\left(\left|\frac{\sqrt{x}}{3}\right|^{3}+\left|\frac{\sqrt{y}}{3}\right|^{3}+\left|\frac{\sqrt{z}}{2}-\frac{\sqrt{x}}{2}\right|^{3}\right) \\
\leq \frac{4}{25}\left(\left|\frac{\sqrt{x}}{3}\right|^{3}+\left|\frac{\sqrt{y}}{3}\right|^{3}+\left|\frac{\sqrt{z}}{2}-\frac{\sqrt{x}}{2}\right|^{3}\right) \\
\leq \frac{1}{\left.\left(\left|\frac{\sqrt{z}}{2}-\frac{\sqrt{x}}{2}\right|^{2}\right)+2\right)^{2}}\left(\left|\frac{\sqrt{x}}{3}\right|^{3}+\left|\frac{\sqrt{y}}{3}\right|^{3}+\left|\frac{\sqrt{z}}{2}-\frac{\sqrt{x}}{2}\right|^{3}\right) \\
=\alpha(G(g x, g y, g z)) \int_{0}^{(G(g x, f x, g x))} \phi(t) d t+\beta(G(g x, g y, g z)) \int_{0}^{(G(g y, f y, g x))} \phi(t) d t \\
+\gamma(G(g x, g y, g z)) \int_{0}^{(G(g x, g y, g z))} \phi(t) d t .
\end{gathered}
$$

Case 3. If $x \in\left[0, \frac{1}{2}\right)$ and $y, z \in\left[\frac{1}{2}, 1\right]$, then

$$
\begin{gathered}
\int_{0}^{G(f x, f y, f z)} \phi(t) d t=(G(f x, f y, f z))^{3} \\
=\left|\frac{\sqrt{x}}{12}-\frac{\sqrt{z}}{6}\right|^{\beta}=\frac{1}{27}\left|\frac{\sqrt{x}}{4}-\frac{\sqrt{z}}{2}\right|^{\beta} \\
\leq \frac{4}{25}\left(\left|\frac{\sqrt{x}}{6}\right|^{\beta}+\left|\frac{\sqrt{x}}{4}-\frac{\sqrt{y}}{2}\right|^{3}+\left|\frac{\sqrt{x}}{4}-\frac{\sqrt{z}}{2}\right|^{3}\right) \\
\leq \frac{1}{\left(\mid\left(\frac{\sqrt{x}}{4}-\left.\frac{\sqrt{z}}{2}\right|^{2}\right)+2\right)^{2}}\left(\left|\frac{\sqrt{x}}{6}\right|^{3}+\left|\frac{\sqrt{x}}{4}-\frac{\sqrt{y}}{2}\right|^{3}+\left|\frac{\sqrt{x}}{4}-\frac{\sqrt{z}}{2}\right|^{\beta}\right) \\
=\alpha(G(g x, g y, g z)) \int_{0}^{(G(g x, f x, g x))} \phi(t) d t+\beta(G(g x, g y, g z)) \int_{0}^{(G(g y, f y, g x))} \phi(t) d t \\
+\gamma(G(g x, g y, g z)) \int_{0}^{(G(g x, g y, g z))} \phi(t) d t .
\end{gathered}
$$

Clearly all conditions of Theorem 3.8 are satisfied. Thus $f$ and $g$ have a unique common fixed point which is 0 .

\section{Remarks}

- $\quad$ Corollary 3.2 and Corollary 3.3 are the results of H (Ayadi, 2012).

- $\quad$ Corollary 3.3 and Corollary 3.11 generalize the results of respectively (Liu et al., 2011; Liu et al., 2014b). 


\section{References}

[1] Abbas, M., Khan, A.R., Nazir, T., (2011). Coupled common fixed point results in two

generalized metric spaces. Applied Mathematics and Computation, 217(13): 6328-6336.

[2] Aliouche, (2003). A common fixed point theorem for weakly compatible mappings in symmetric spaces.

[3] Agarwal, R.P., ORegan, D., Shahzad, N., (2004). Fixed point theorems for generalized contractive maps of Mei-Keeler type. Mathematise Nachrichten, 276(1): 3-12.

[4] Ayadi, H., (2012). A common fixed point of integral type contraction in Generalized Metric space. Journal of Advanced Mathematical Studies, 5: 111-117.

[5] Berinde, V., (2003). On the approximation of fixed points of weak contractive mappings.

Carpathian Journal of Mathematics, 19(1): 7-22.

[6] Boyd, D.W., Wong, J.S.W., (1969). On nonlinear contractions. Proceedings of the American Mathematical Society, 20: 458-464.

[7] Branciari, (2002). A fixed-point theorem for mapping satisfying a general contractive condition of integral type. International Journal of Mathematics and Mathematical Sciences, 29(9): 531-536.

[8] Ciric, L.J.B., (1974). A generalization of Banach contraction principle. Proceedings of the

American Mathematical Society, 45: 267-273.

[9] Djoudi, A., Merghadi, F., (2008). Common fixed point theorems for maps under a contractive

condition of integral type. Journal of Mathematical Analysis and Applications, 341(2): 953-960.

[10] Khojesteh, F., Goodarzi, Z., and Razani, A., (2010). Some fixed point theorem of integral type contraction in cone metric spaces. Fixed Point Theory and Applications, 2010: 1-13.

[11] Liu, Z., Xu, B., Kang, S.M., (2014a). Two fixed point Theorems of mappings satisfying contractive inequalities of integral type. International journal of pure and applied mathematics, 90: 85-100.

[12] Liu, Z., kang, S.M., (2000). Existence and uniqueness of solutions for two classes of functional equations arising in dynamic programming. Scientific Annals of the Alexandru Ioan Cuza University of Iasi, 46(2): 3-24.

[13] Liu, Z., Kang, S.M., and Cho, S.Y., (2011). Fixed point theorems for mapping satisfying

contractive condition of integral type and application. Fixed point theory and applications,

64:1-18.

[14] Liu, Z., Han, Y., Kang, S.M., and Ume, J. S., (2014b). Common fixed point theorems for weakly compatible mappings satisfying contractive conditions of integral type. Fixed Point Theory and Applications, 132: 1687-1812.

[15] Manro, S., Bhatic, S., Kumar, S., and Vetro, C., (2013). A common fixed point theorem for two

weakly compatible pairs in G-metric spaces using the property E.A. Fixed point theory and Applications, 41.

[16] Mustafa, Z., Sims, B., (2006). A new approach to generalized metric spaces. Journal of nonlinear and convex analysis, 7: 289-297.

[17] Nadlar, S.B., (1969). Multivalued contraction mappings. Pacific Journal of Mathematics, 30: 475-488.

[18] Rhoades, B.E., Two fixed point theorem for mapping satisfying a general contractive condition of integral type.
International Journal of Mathematics and Mathematical Sciences, 63: 4007-4013.

[19] Satisfying a contractive condition of integral type, Journal of Mathematical Analysis and

Applications, 322, 796-802, (2006).

[20] Sintunavarat and Kumam, (2012). Generalized common fixed point theorems in complex valued metric spaces and applications. Journal of Inequalities and Applications, 84: 63286336.

[21] Suuki, T., (2006). Meir-Keeler contraction of integral type are still Meir-Keeler contraction.

International Journal of Mathematics and Mathematical Sciences, 16.

[22] Suzuki, T., (2008). A generalized Banach contraction principle that characterizes metric

Completeness. Proceedings of the American Mathematical Society, 136(5): 1861-1869.

[23] Zamfrescu, T., (1972). Fixed point theorems in metric spaces. Archivum Mathematicum (Basel),

23: 292-298. 